\title{
An Advanced Technology Performance Measure Method for System
}

\author{
Zhang $\mathrm{Lu}^{1,2,{ }^{*}}$, Lv Meilei ${ }^{1}$, Wang Hailun ${ }^{1}$ and Zhang Yalong ${ }^{1}$ \\ ${ }^{I}$ College of Electrical and Information Engineering, Quzhou University, Quzhou, 324000, China \\ ${ }^{2}$ School of Computer, Wuhan University of Technology, Wuhan, 430070, China
}

\begin{abstract}
Arming at TRMs only accounts for the achieved performance instead of both the achieved and unmet performance, so it is impossible to reflect the aggregated risk. This paper firstly extends the technology category in TPMs. A new kind of technology is added to the traditional TRMs and the calculating formula is given. Secondly, the proposed TPRM is based on two concepts: the unmet performance and the degree of difficulty with the unmet performance. At last, two applications are given to prove the availability of the method in the paper.
\end{abstract}

Keywords: Technical performance requirements (TPR), Technical performance measure (TPM), Degree of difficulty(DD), Technology performance risk measure (TPRM), System.

\section{INTRODUCTION}

Technical Performance Measures (TPMs) are defined and evaluated on how well a system is achieving its performance requirement indicators. The TPM can provide the information that it is achieving the intended target with the development progress of the system performance, but, these TPM are just a response of the system's single performance parameters. Comprehensive individual indicators obtained the technical performance of the integrated metrics which reflects the overall state.

In addition, TPR is a measure of technology to achieve performance requirements. It is a measure of the past, without considering the difficulty of application technology. As TPM does not consider the risk of completion of the remaining performance requirements, TPM needs to be further improved, need to adopt a more reasonable metric to mark the integrated risk [1-4].

Firstly, the original TPM of technical performance indicators is expanded for 3 types, and given the respective TPM formula; Second, Technology performance risk measure (TPRM) is proposed, and the rapid discriminating map is given.

\section{THE ESTABLISHMENT OF TECHNICAL PER- FORMANCE MEASURE}

\subsection{Traditional Technical Performance Measure}

The classical TPM is to assess the situation of the system's technical performance in one aspect, and then obtains the performance measure of the system through different indicators, to have a global understanding of the progress of the whole system. In order to meet equipment requirements for war, some requirements of the technical performance of the system require its value to be higher and some require its value to be lower. According to this feature, this paper divides technical indicators into two categories [58]. The lower value classified as Class A, on the contrary for the Class B. Although some of the indicators required the higher the better or the lower the better, but they can not be infinitely high or infinitely low, so we only require meeting the performance requirements of the tasks. Class A of technical performance may require a threshold to meet the mission requirements. Class $\mathrm{A}$ is more than the threshold means it meets the requirements; Similarly, Class B is less than the threshold means it meets the requirements. As shown in Figs. $(\mathbf{1}, \mathbf{2})$.

\subsection{Modified Technical Performance Measure}

In addition to the two technical measures above, there is another case: technical performances for system meet the performance requirements within the specified range, higher than the upper limit or below the lower limit does not meet the requirements, categorized as Class C. This is similar to the pulse of the body, healthy body requires a certain range in pulse rate, and pulses too fast or too slow per minute are unhealthy signs. Provides Class $\mathrm{C}$ to meet the upper threshold and lower threshold for task requirement, which can be given in accordance with task requirements, Class $\mathrm{C}$ between the upper threshold and the lower threshold value is described to meet the requirements, shown in Fig. (3).

The horizontal axis in Fig. (3) the same as Fig. (1) and (2), $V_{U p-t h r e s}$ means upper threshold, $V_{\text {Low-thres }}$ means lower threshold.

Since there is no uniform standard and units of measurement between each TPM, and the original data can not be 


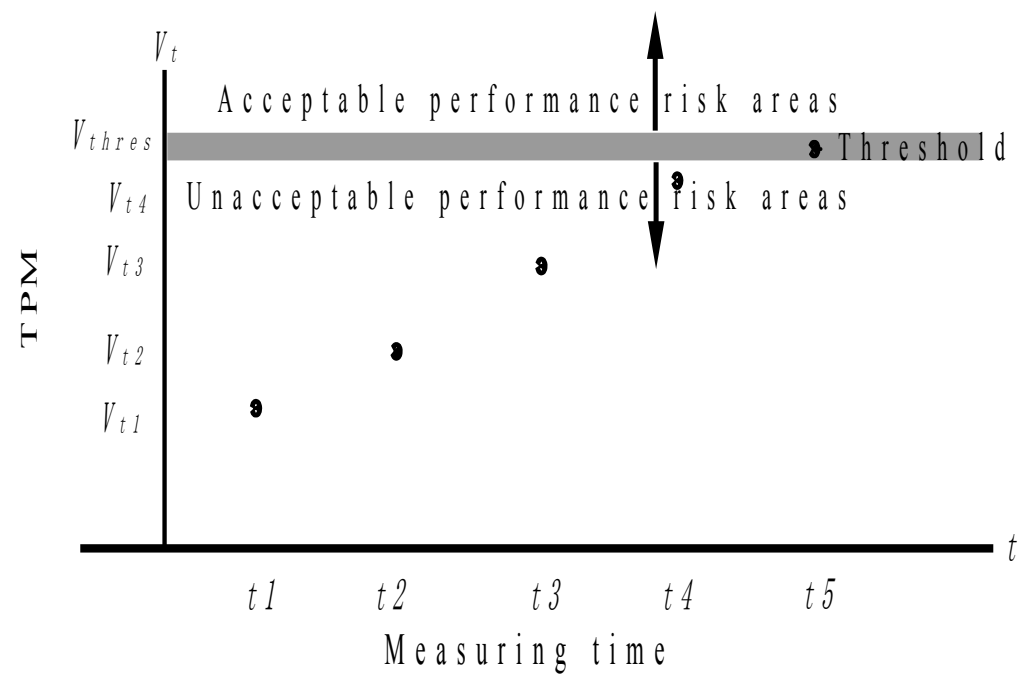

Fig. (1). Class A specifications.

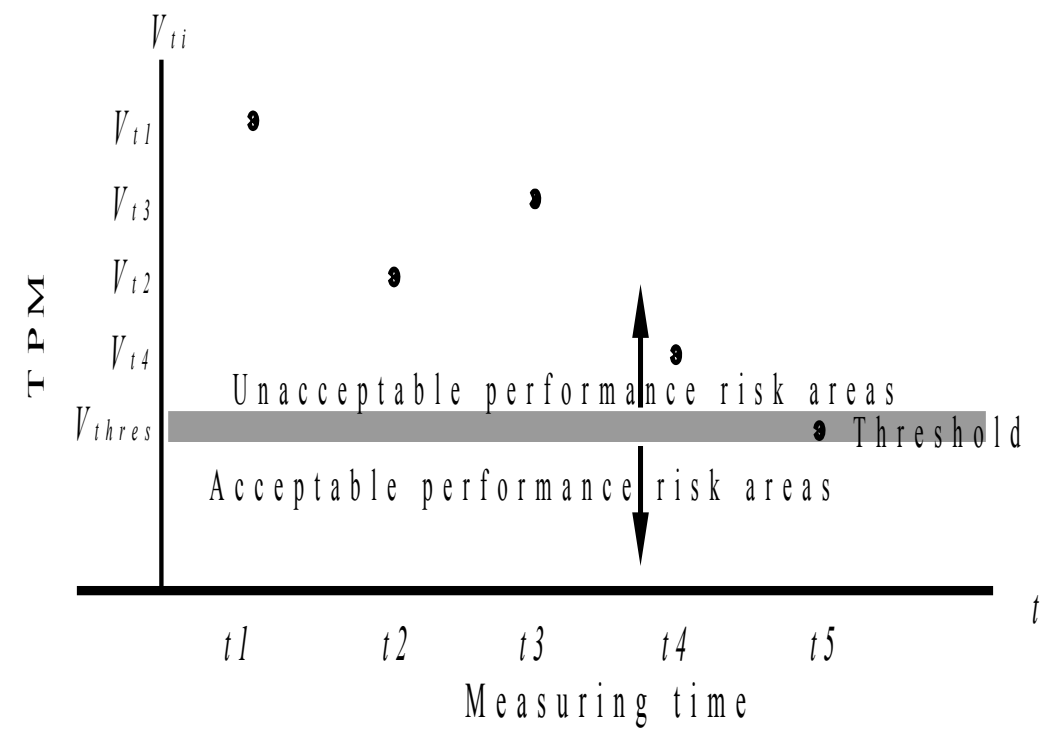

Fig. (2). Class B specifications.

compared with each other directly, the original data needs to be normalized. For the 3' specifications of the classes, we proposed the following normalization methods in this paper.

$V_{t i, A j}$ means Class A's TPM with item $\mathrm{j}$ in $t i$ measuring time, $V_{\text {thres }, A j}$ means the threshold for Class A's TPM with item $\mathrm{j}, v_{t i, A j}$ means Class A's TPM with item $\mathrm{j}$ in $t i$ measuring time which after normalization of.

$$
\begin{aligned}
V_{t i, A j} & =\max \left\{V_{t i, A j}, V_{\text {thres }, A j}\right\} / V_{\text {thres }, A j} \\
& =\max \left\{V_{t i, A j} / V_{\text {thres }, A j}, \quad 1\right\} \\
& =\max \left\{\left(V_{\text {thres } A j}-V_{\text {thres }, A j}+V_{\text {ti, } A j}\right) / V_{\text {thres }, j}, \quad 1\right\} \\
& =\max \left\{\left[1+\left(V_{t i, A j}-V_{\text {thres }, A j}\right) / V_{\text {thres }, A j}\right], \quad 1\right\} \quad(\geq 1)
\end{aligned}
$$

For Class B, $V_{t i, B k}$ means Class B's TPM with item k in $t i$ measuring time, $V_{\text {thres }, B k}$ means the threshold for Class B's TPM with item $\mathrm{k}, v_{t i, B k}$ means Class B's TPM with item $\mathrm{k}$ in $t i$ measuring time which after normalization of.

$$
\begin{aligned}
v_{t i, B k} & =\min \left\{V_{t i, B k}, V_{\text {thres }, B k}\right\} / V_{\text {thres }, B k} \\
& =\min \left\{V_{t i, B k} / V_{\text {thres }, B k}, \quad 1\right\} \\
& =\min \left\{\left[1-\left(V_{\text {thres }, B k}-V_{t i, B k}\right) / V_{\text {thres }, B k}\right], \quad 1\right\} \quad(\leq 1)
\end{aligned}
$$

The same as Class C, $V_{t i, C l}$ means Class C's TPM with item 1 in $t i$ measuring time, $V_{U p-t h r e s, C l}$ means the upper 


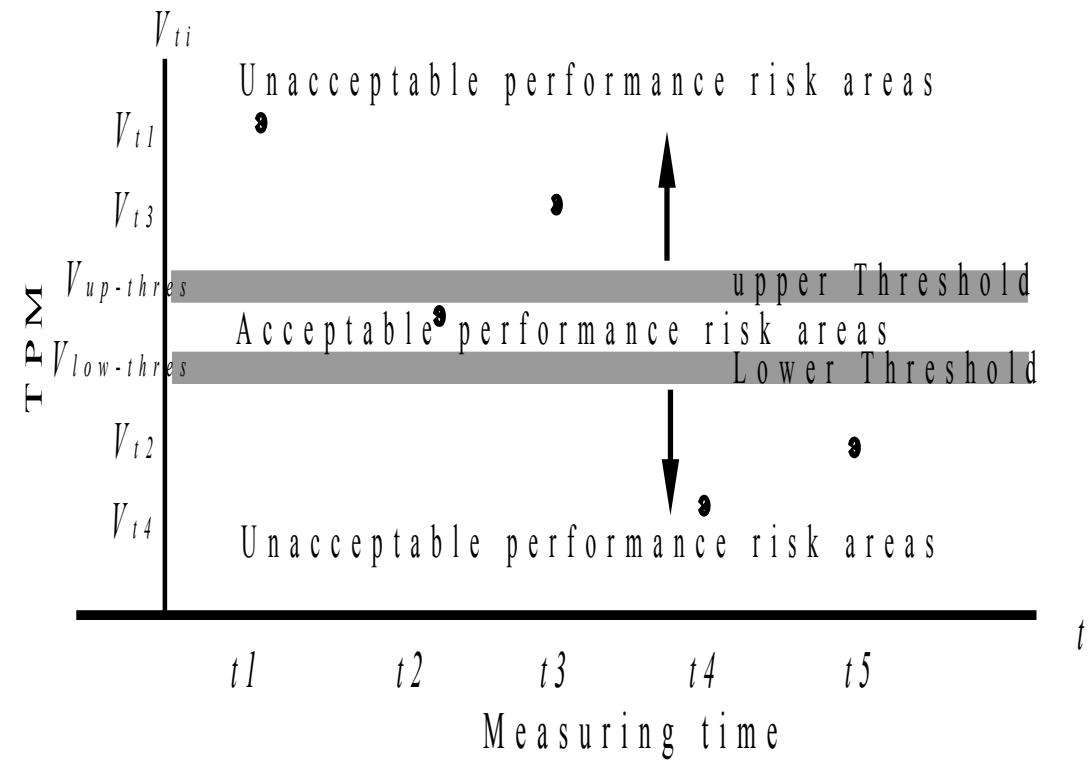

Fig. (3). Class C specifications.

threshold for Class C's TPM with item 1, $V_{\text {Low-thres, } C l}$ means the lower threshold for Class C's TPM with item 1, $v_{t i, C l}$ means Class C's TPM with item 1 in $t i$ measuring time which after normalization of.

$v_{t i, C j}=\left\{\begin{array}{lc}\max \left\{V_{t i, C j}, V_{U p-\text { thres }, C j}\right\} / V_{U p-\text { thres }, C j} & V_{t i, C j}>V_{U p-t h r e s, C j} \\ 1 & V_{\text {Low-thres }, C j} \leq V_{t i, C j} \leq V_{U p-t h r e s, C j} \\ \min \left\{V_{t i, C j}, V_{\text {Low-thres }, C j}\right\} / V_{\text {Low-thres }, C j} & V_{t i, C j}<V_{\text {Low-thres }, C j}\end{array}\right.$

For combinations of all normalized TPM to unity values reflect the different techniques, Class $\mathrm{A}$ and $\mathrm{C}$ will be normalized to range $[0,1]$

$$
\begin{aligned}
u_{t i, A j} & =1 /\left[\max \left\{V_{t i, A j}, V_{\text {thres }, A j}\right\} / V_{\text {thres }, A j}\right] \\
& =1 / v_{t i, A j} \quad(\leq 1)
\end{aligned}
$$

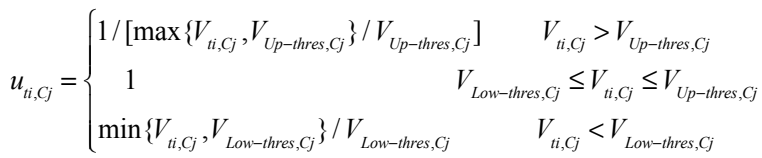

In index comprehensive, using the regular systematic reviews modeling, according to the importance of each TPM in system requirements, combined weight coefficients such as expert ratings and AHP methods for determining the weight of each indicator, then comprehensive. The result as the comprehensive technical performance metrics $T P M_{\text {ti,all }}$.

Assumed $j=1,2, \cdots, m, \quad k=1,2, \cdots, n, \quad l=1,2, \cdots, p$, for Class A, comprehensive technical performance metric is calculated as follows:

$$
T P M_{t i, A}=\left(w_{A 1} u_{t j, A 1}+w_{A 2} u_{t j, A 2}+\cdots+w_{A m} u_{t j, A m}\right) / W_{A}
$$

$w_{A j}(j=1,2, \ldots, m)$ means the weight of $A_{j}$, and $W_{A}=w_{A 1}+w_{A 2}+\cdots+w_{A m}$.

Similarly:

$T P M_{t j, B}=\left(w_{B 1} v_{t j, B 1}+w_{B 2} v_{t j, B 2}+\cdots+w_{B m} v_{t j, B n}\right) / W_{B}$

$W_{B}=w_{B 1}+w_{B 2}+\cdots+w_{B m}$

$T P M_{t j, C}=\left(w_{C 1} u_{t j, C 1}+w_{C 2} u_{t j, C 2}+\cdots+w_{C m} u_{t j, C n}\right) / W_{C}$

$W_{C}=w_{C 1}+w_{C 2}+\cdots+w_{C m}$

Considered the weights $W_{A} 、 W_{B} 、 W_{C}$ of Class A、B、 $\mathrm{C}$, there are:

$$
\begin{aligned}
& T P M_{t j, A l l}=\left(W_{A} T P M_{t j, A}+W_{B} T P M_{t j, B}+W_{C} T P M_{t j, C}\right) / W \\
& W=W_{A}+W_{B}+W_{C}
\end{aligned}
$$

\section{THE ESTABISHIMENT OF TECHNICAL PER- FORMANCE RISK MEASURE}

\subsection{Degree of Difficulty}

In considering the technology to achieve performance requirements, we must pay attention to the degree of difficulty which is to complete the remaining technical performance requirements. It is proposed that the concept of degree of difficulty that is expected for risk measurement from lowrisk to high-risk, can be considered a failure probability to achieve performance goals, the degree of difficulty ranges from $[0,1]$. DD expressed degree of difficulty, means, the 
Table 1. The concept of technical difficulties index.

\begin{tabular}{|c|c|c|}
\hline Degree of Difficulty & Risk Level & DD Value \\
\hline \hline Minimum degree of difficulty & Success & 0.0 \\
\hline 1 & Very low risk & 0.1 \\
\hline 2 & Low risk & 0.3 \\
\hline 3 & Medium risk & 0.5 \\
\hline 4 & High risk & 0.7 \\
\hline 5 & Very high risk, need basic technological breakthroughs & 0.9 \\
\hline Maximum degree of difficulty & Failure & 1.0 \\
\hline
\end{tabular}

degree of difficulty that the system is able to complete the remaining requirements. Reference Mankins's [9] concept of $\mathrm{R} \& \mathrm{D}$ difficulty, Following Table 1 shows the division of DD.

Technical difficulties index expressed the failure probability of completing the remaining performance requirements, High degree of difficulty expressed to complete the unfinished performance requirements have high risk and high probability of failure; Low degree of difficulty expressed to complete the unfinished performance requirements have low risk and low probability of failure. The minimum level of technical difficulties expressed have no risk, the maximum level expressed technical solutions can not achieve the remaining performance requirements.

\subsection{Technical Performance Risk Measure}

Technical performance risk measure is the function of technical performance measure and degree of difficulty, it can be expressed with the following formula as follow:

$T P R M=f(T P M, D D)$

According to the limit of TPM and DD, the boundary conditions for TPRM can be given:

1) The degree of difficulty complete the remaining performance requirement to 0, TPRM can represent the remaining performance requirements;

2) The degree of difficulty complete the remaining performance requirement to 1 , that is impossible to complete the remaining performance requirements, the performance requirements system is absolutely unrealizable, TPRM = 1;

3) When the TPM fully meets all performance requirements, TPRM $=0$, is almost totally without any risk at this time;

4) Does not implement any TPM, that is, when TPM is 0 , does not meet any performance requirements, clearly, technical performance is definitely the highest risk at this time: TPRM $=1$;
According to the four conditions above, the following formulas can be obtained:

$\lim _{D D \rightarrow 0} T P R M=1-T P M$

$\lim _{D D \rightarrow 1} T P R M=1$

$\lim _{T P M \rightarrow 1} T P R M=0$

$\lim _{T P M \rightarrow 0} T P R M=1$

According to the above four conditions can be calculated for TPRM:

$T P R M=\frac{1-T P M}{1-T P M \times D D^{n}}$

Where $\mathrm{n}$ can take rational numbers, the general calculations take $\mathrm{n}=1$.

For different DD, correspondence between TPM and TPRM is shown in Fig. (4). Only when DD is 0 , there exist a linear relation between TPM and TPRM, TPM is just TPRM's special form.

For different TPM, correspondence between DD and TPM is shown in Fig. (5). When TPM is small, the impact of DD is less. When TPM is large, with DD increasing, TPRM increases.

Calculated the contour TPRM between the different degree of difficulty and technical performance measure through the formula 18, as shown in Fig. (6), thus the method of rapid assessment by technical performance risk measurement is given in different combinations of DD and TPM, as shown in Fig. (7).

Under combination of different type of technology the technical performance risk measure can be calculated, we get technical performance risk measurement formula as:

$$
\begin{aligned}
& \operatorname{TPRM}_{t i, A l l}=\left(W_{A} T P R M_{t j, A}+W_{B} T P R M_{t i, B}+W_{C} T P R M_{t, C}\right) / W \\
& W=W_{A}+W_{B}+W_{C}
\end{aligned}
$$




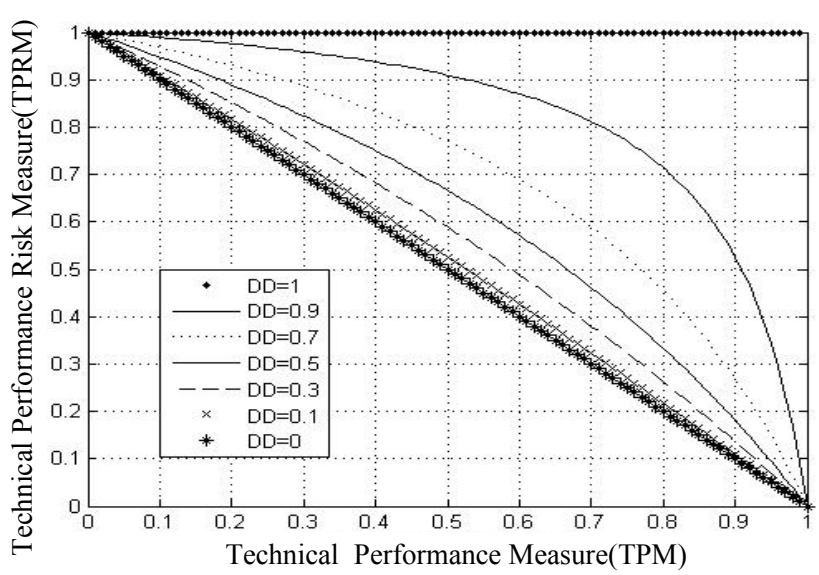

Fig. (4). Relation between TPM and TPRM for different DD.

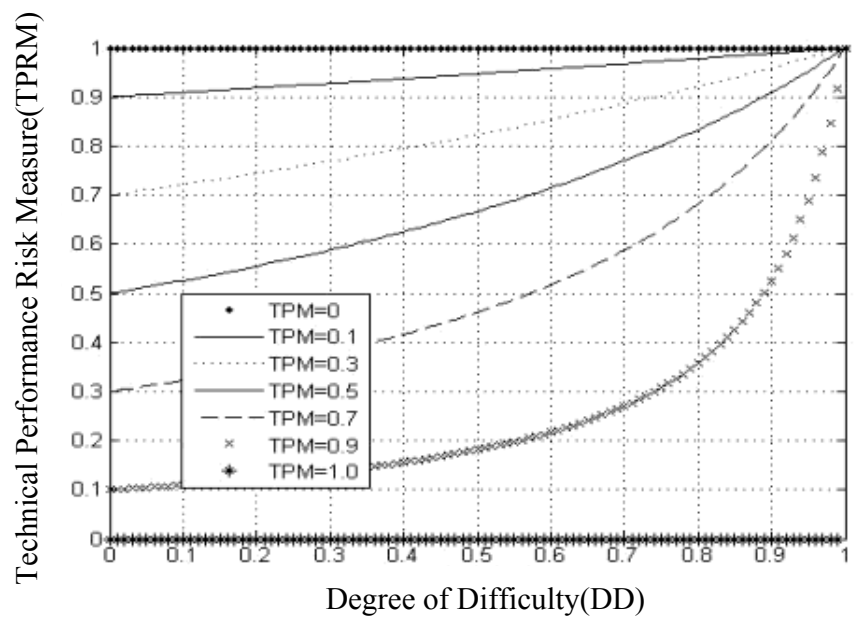

Fig. (5). Relation between DD and TPRM for different TPM.

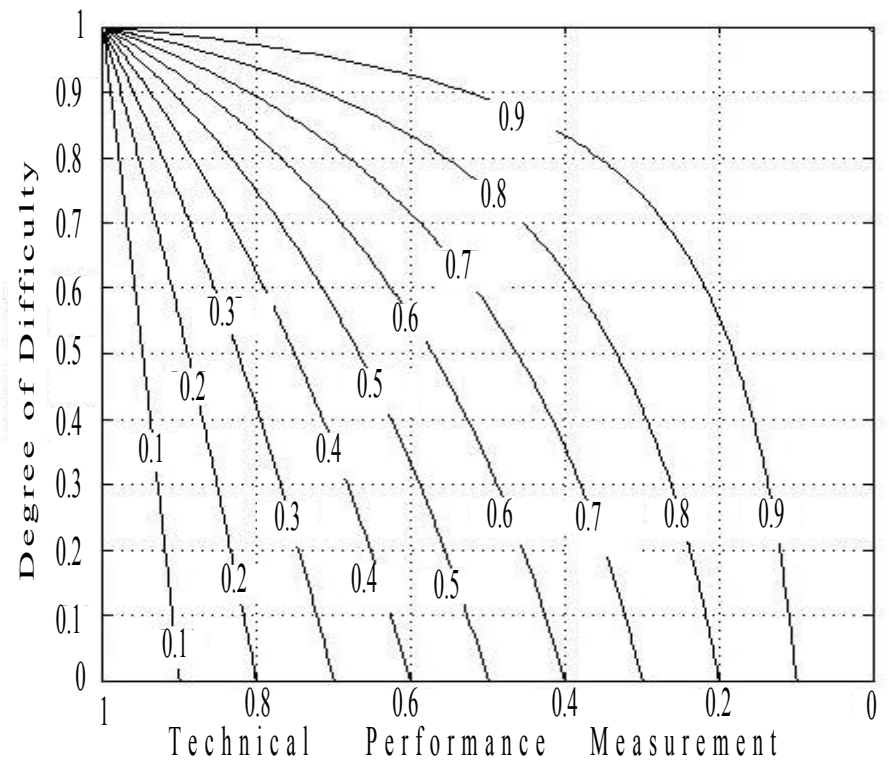

Fig. (6). TPRM contour between DD and TPM. 


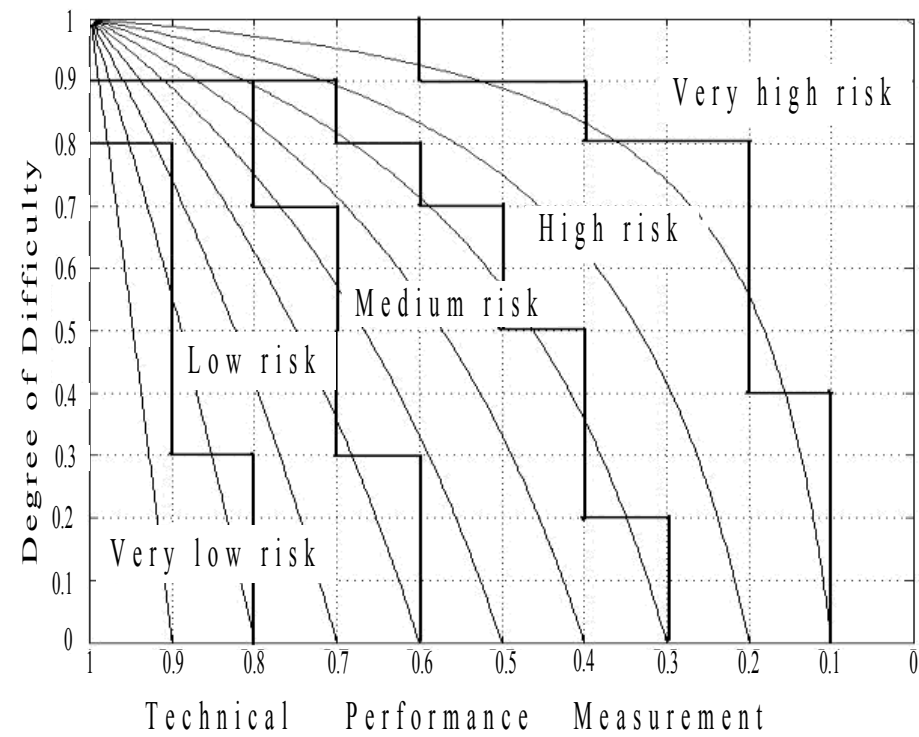

Fig. (7). TPRM allocation of risk between DD and TPM.

Table 2. TPM index calculation for class A technology.

\begin{tabular}{|c|c|c|c|c|c|}
\hline Metric Time & & MTBF & Reaction Time & Interval & $T P M_{A, \text { all }}$ \\
\hline & Weights & 1.0 & 1.0 & 3.0 & \\
\hline & Threshold & 0.5 & 5 & 10 & \\
\hline $\mathrm{t} 1$ & Vt1,A、vt1,A & 1、 0.5 & $11 、 0.456$ & $30 、 0.33$ & 0.371 \\
\hline $\mathrm{t} 2$ & $\mathrm{Vt} 2, \mathrm{~A} 、 \mathrm{vt} 2, \mathrm{~A}$ & $0.9 、 0.56$ & $10 、 0.5$ & $25 、 0.4$ & 0.452 \\
\hline $\mathrm{t} 3$ & $\mathrm{Vt} 3, \mathrm{~A} 、 \mathrm{vt} 3, \mathrm{~A}$ & $0.8 、 0.625$ & $8.5 、 0.588$ & $21,0.476$ & 0.528 \\
\hline $\mathrm{t} 4$ & $\mathrm{Vt} 4, \mathrm{~A} 、 \mathrm{vt} 4, \mathrm{~A}$ & $0.7 、 0.71$ & 7、 0.714 & $15 、 0.667$ & 0.685 \\
\hline t5 & Vt5,A、vt5,A & $0.6 、 0.83$ & $6.5 、 0.769$ & $12 、 0.833$ & 0.82 \\
\hline t6 & Vt6,A、vt6,A & $0.5 、 1$ & 5,1 & 9、 1 & 1 \\
\hline
\end{tabular}

Determining the results of the TPRM by the risk determines matrix for TPRM in Fig. (4), we get the degree of system technical performance risk measures.

\section{APPLICATION EXAMPLE}

\subsection{One Example: the Application of Modify Technical Performance Measure}

From literature [10], combining with wireless sensor networks and applying the above methods to assess technical performance risk, measures the TPM indicators of the system which can be concluded into the following categories:

Class A index: MTBF (hours), reaction time (minutes), interval (seconds)

Class B index: MTTR (hours), precision (\%)
Class $\mathrm{C}$ index: Firing frequency $(\mathrm{HZ})$

Various types of indicators are defined by the respective threshold, the original data and the corresponding results are in Table 2-4.

From the formula, obtain the value of the technical performance measure for different times, as shown in Fig. (8), which shows the extent required to achieve the desired system performance for different time stages.

\subsection{Second Example: the Application of Technical Per- formance Risk Measure}

In a system, with two alternative technologies (technology A and technology B) to choose from, decision makers need to choose which of the two can be applied in the system. Both technology A and technology B can achieve 
Table 3. TPM index calculation for class B technology.

\begin{tabular}{|c|c|c|c|c|}
\hline Metric Time & & MTTR & Accuracy & $T P M_{B, a l}$ \\
\hline & Weights & 1.0 & 2.0 & \\
\hline & Threshold & 60 & $85 \%$ & \\
\hline $\mathrm{t} 1$ & Vt1,B、vt1,B & $25 、 0.417$ & $50 \%, 0.589$ & 0.531 \\
\hline $\mathrm{t} 2$ & Vt2,B、vt2,B & $30 、 0.5$ & $56 \%, 0.659$ & 0.606 \\
\hline $\mathrm{t} 3$ & Vt3,B、vt3,B & $33 、 0.55$ & $65 \%, 0.765$ & 0.693 \\
\hline $\mathrm{t} 4$ & Vt4,B、vt4,B & $45 、 0.75$ & $75 \%, 0.882$ & 0.838 \\
\hline t5 & Vt5,B、vt5,B & 55, 0.917 & $80 \%, 0.941$ & 0.933 \\
\hline t6 & Vt6,B、vt6,B & $63 、 1$ & $88 \% 、 1$ & 1 \\
\hline
\end{tabular}

Table 4. TPM index calculation for class $C$ technology.

\begin{tabular}{|c|c|c|c|}
\hline Metric Time & & Engine Shock Frequency & $T P M_{C, \text { all }}$ \\
\hline & Weights & 0.5 & \\
\hline & Threshold & $60-66$ & \\
\hline $\mathrm{t} 1$ & $\mathrm{Vt1}, \mathrm{C} 、 \mathrm{vt} 1, \mathrm{C}$ & $90 、 0.733$ & 0.733 \\
\hline $\mathrm{t} 2$ & $\mathrm{Vt} 2, \mathrm{C} 、 \mathrm{vt} 2, \mathrm{C}$ & $81 、 0.815$ & 0.815 \\
\hline $\mathrm{t} 3$ & $\mathrm{Vt} 3, \mathrm{C} 、 \mathrm{vt} 3, \mathrm{C}$ & $50 、 0.833$ & 0.833 \\
\hline $\mathrm{t} 4$ & $\mathrm{Vt} 4, \mathrm{C} 、 \mathrm{vt} 4, \mathrm{C}$ & $55 、 0.917$ & 0.917 \\
\hline $\mathrm{t} 5$ & $\mathrm{Vt5}, \mathrm{C} 、 \mathrm{vt5}, \mathrm{C}$ & $68 、 0.97$ & 0.97 \\
\hline t6 & Vt6,C、vt6,C & $62 、 1$ & 1 \\
\hline
\end{tabular}

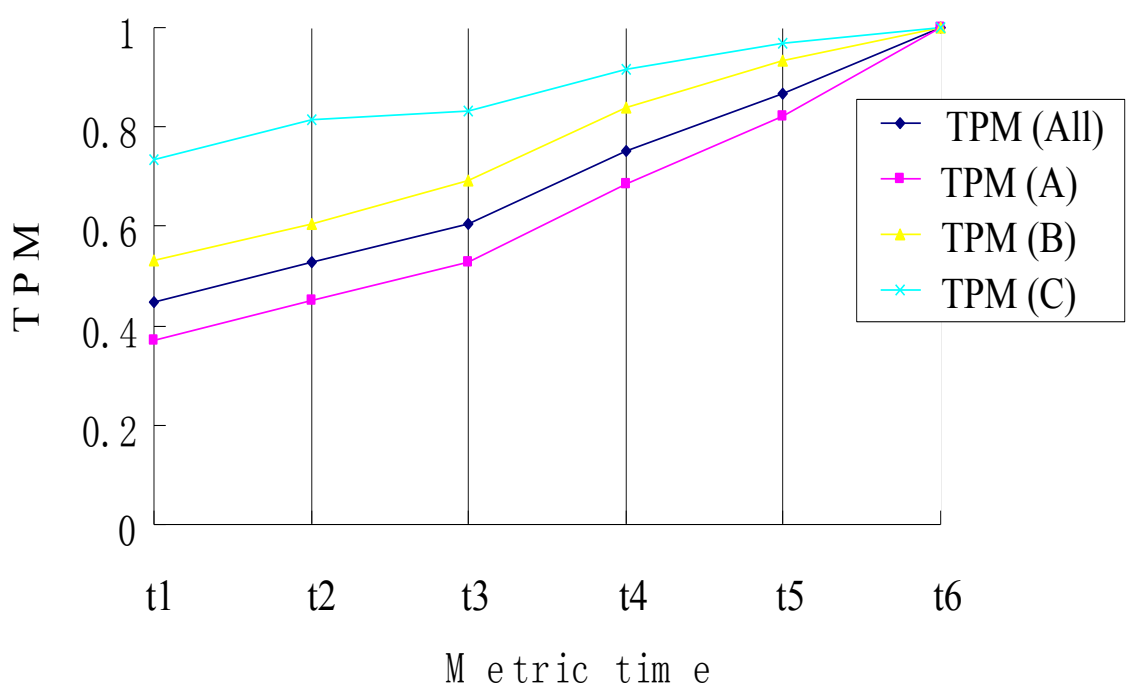

Fig. (8). The TPM value of different measurement time.

technical performance measure of $60 \%$, and technology B's TPM is slightly higher than technology A's. The different applications of technology A and technology B are: technology A, use of Commercial-off-the-shelf components, mainly in the 70's to 80's technology; Technology B is a new technology that requires accurate processing and sophisticated test system. The degree of difficulty (DD) of the technology $\mathrm{A}$ is much less than technology $\mathrm{B}$, as shown in Table $\mathbf{5}$. 
Table 5. TPRM computation of a certain system alternative technologies.

\begin{tabular}{|c|c|c|c|}
\hline Technical Program & TPM Value has been Reached & DD of Remaining TPM & TPRM \\
\hline \hline A & 0.61 & 0.2 & 0.44 \\
\hline B & 0.73 & 0.7 & 0.55 \\
\hline
\end{tabular}

Although the technology B can achieve a higher TPM value, because of the high degree of difficulty in completing the remaining TPM, so the technical performance risk is also higher than $\mathrm{A}$, in other words, the failure rate of technical $\mathrm{B}$ is more than technical $\mathrm{A}$.

\section{CONCLUSION}

This paper presents a method to improve the technical performance measurement, through two examples, the results show that the measure system can fully reflect the system's overall risk, can assist decision-making, and is reasonable and scientific in the practical application.

\section{CONFLICT OF INTEREST}

The author confirms that this article content has no conflict of interest.

\section{ACKNOWLEDGEMENTS}

This work was partially supported by National Nature Science Fund of China (NSFC) (Grant 1403229), Zhejiang Provincial Nature Science Foundation of China (Grant No. LY13F030011), Quzhou Science and Technology Project
(2014Y006) and Quzhou University middle-aged and young backbone teachers funded project (XNZQN201312).

\section{REFERENCES}

[1] C. Jiang-Ning, W. Jun, Y. Bo, and H. Tao, "Adaptive failure detection in web application server," Journal of Software vol.16, no.11, pp. 1929-1938, 2005.

[2] L. Lei, "Research on Performance Optimization Techniques in Fault Tolerant Distributed Systems," HuNan: ChangSha. College of Computer: National University of Defense Technology, 2007.

[3] P.P. Narasimhan, Causes of Failure in Web Applieations. Carnegie Mellon University Parallel Data Lab Technieal Report, CMU-PDL05-109, December 2005, pp. 225-229.

[4] F. Cristian, "Understanding fault-tolerant distributed systems," Communications of the ACM, vol. 34, no. 2, pp. 57-58, 1991.

[5] P. R. Garvey, and C. -C. Cho, "An index to measure a system's performance risk," Acquisition Review Quarterly, vol. 10, no. 2, pp. 189-199, 2003.

[6] Q. X. -Tian, and L. Jian-Wei, "A system evaluation method for combined technical performance measure based On TPM," Control and Automation Publication Group, vol. 22, pp. 90-93, 2006.

[7] D. K. Pradhan, Fault-Tolerant Computer System Design, Prentice Hall: PTR, June 1996, pp. 994-1008.

[8] J. Wu, Distributed System Design, CRC Press: LLC, 1999, pp. 587593.

[9] J. C. Mankins, Research \& Development Degree of Difficulty (R\&D3), White Paper, NASA Head quarters, 1998, pp. 112-115.

[10] H. Jing, and W. Gang, "Research on model base system of C3i system simulation," Computer Engineering vol. 29, no. 4, pp. 5456, 2003.

(C) Lu et al.; Licensee Bentham Open.

This is an open access article licensed under the terms of the Creative Commons Attribution Non-Commercial License (http://creativecommons.org/licenses/by-nc/3.0/) which permits unrestricted, non-commercial use, distribution and reproduction in any medium, provided the work is properly cited. 